\title{
Quantitative analysis of the mouth opening movement of temporomandibular joint disorder patients according to disc position using computer vision: a pilot study
}

\author{
Kug Jin Jeon ${ }^{1 \#} \wedge$, Young Hyun $\mathrm{Kim}^{1 \# \wedge}$, Eun-Gyu Ha ${ }^{1 \wedge}$, Han Seung Choi ${ }^{1 \wedge}$, Hyung-Joon Ahn ${ }^{2} \wedge$, Jeong \\ Ryong Lee ${ }^{3} \wedge$, Dosik Hwang ${ }^{1,3,4}$, Sang-Sun Han ${ }^{1,4,5} \wedge$ \\ ${ }^{1}$ Department of Oral and Maxillofacial Radiology, Yonsei University College of Dentistry, Seoul, Korea; ${ }^{2}$ Department of Orofacial Pain and Oral \\ Medicine, Dental Hospital, Yonsei University College of Dentistry, Seoul, Korea; ${ }^{3}$ Department of Electrical and Electronic Engineering, Yonsei \\ University, Seoul, Korea; ${ }^{4}$ Clinical Imaging Data Science (CCIDS), Yonsei University College of Medicine, Seoul, Korea; ${ }^{5}$ Department of Computer \\ Science, Yonsei University, Seoul, Korea
}

Contributions: (I) Conception and design: KJ Jeon, YH Kim, SS Han; (II) Administrative support: SS Han; (III) Provision of study materials or patients: KJ Jeon, YH Kim, HJ Ahn, D Hwang, SS Han; (IV) Collection and assembly of data: EG Ha, HS Choi, JR Lee; (V) Data analysis and interpretation: KJ Jeon, YH Kim, EG Ha, HS Choi, JR Lee; (VI) Manuscript writing: All authors; (VII) Final approval of manuscript: All authors.

\#These authors contributed equally to this work.

Correspondence to: Sang-Sun Han, DDS, PhD. Department of Oral and Maxillofacial Radiology, Yonsei University College of Dentistry, 50-1 Yonseiro, Seodaemun-gu, Seoul 03722, Korea. Email: sshan@yuhs.ac.

Background: Temporomandibular joint disorder (TMD), which is a broad category encompassing
disc displacement, is a common condition with an increasing prevalence. This study aimed to develop an
automated movement tracing algorithm for mouth opening and closing videos, and to quantitatively analyze
the relationship between the results obtained using this developed system and disc position on magnetic
resonance imaging (MRI).
Methods: Mouth opening and closing videos were obtained with a digital camera from 91 subjects, who
underwent MRI. Before video acquisition, an 8.0 -mm-diameter circular sticker was attached to the center of
the subject's upper and lower lips. The automated mouth opening tracing system based on computer vision
was developed in two parts: (I) automated landmark detection of the upper and lower lips in acquired videos,
and (II) graphical presentation of the tracing results for detected landmarks and an automatically calculated
graph height (mouth opening length) and width (sideways values). The graph paths were divided into three
types: straight, sideways-skewed, and limited-straight line graphs. All traced results were evaluated according
to disc position groups determined using MRI. Graph height and width were compared between groups
using analysis of variance (SPSS version 25.0 ; IBM Corp., Armonk, NY, USA).
Results: Subjects with a normal disc position predominantly ( $85.72 \%$ ) showed straight line graphs.
The other two types (sideways-skewed or limited-straight line graphs) were found in $85.0 \%$ and $89.47 \%$
in the anterior disc displacement with reduction group and in the anterior disc displacement without
reduction group, respectively, reflecting a statistically significant correlation $\left(\chi^{2}=38.113\right.$, P<0.001). A
statistically significant difference in graph height was found between the normal group and the anterior disc
displacement without reduction group, $44.90 \pm 9.61$ and $35.78 \pm 10.24$ mm, respectively (P<0.05).

Conclusions: The developed mouth opening tracing system was reliable. It presented objective and

^ ORCID: Kug Jin Jeon, 0000-0002-5862-2975; Young Hyun Kim, 0000-0001-6541-3293; Eun-Gyu Ha, 0000-0002-4436-1751; Han Seung Choi, 0000-0002-7375-2401; Hyung-Joon Ahn, 0000-0001-9669-9781; Jeong Ryong Lee, 0000-0001-7251-2126; Dosik Hwang, 00000002-2217-2837; Sang-Sun Han, 0000-0003-1775-7862. 
quantitative information about different trajectories from those associated with a normal disc position in mouth opening and closing movements. This system will be helpful to clinicians when it is difficult to obtain information through MRI.

Keywords: Artificial intelligence (AI); magnetic resonance imaging (MRI); temporomandibular joint disorder (TMD)

Submitted Jun 15, 2021. Accepted for publication Sep 29, 2021.

doi: 10.21037/qims-21-629

View this article at: https://dx.doi.org/10.21037/qims-21-629

\section{Introduction}

Temporomandibular joint disorder (TMD) is a common condition, with a prevalence of about $40 \%(1,2)$, and the number of patients seeking medical care for TMD is increasing over time (3). Disc displacement is a subtype of TMD (4), for which the most accurate diagnostic tool is magnetic resonance imaging (MRI) as a modality capable of evaluating the position and shape of the disc and the range of condylar head movement $(5,6)$. One of the clinical examinations used to estimate disc displacement is to assess whether there is deviation or deflection during mouth opening movement (7). This movement occurs as a complex series of interrelated three-dimensional rotational and translational activities of both temporomandibular joints (TMJs). Each TMJ cannot function independently. In the early stage of mouth opening, rotation occurs in the inferior cavity of the joint, followed by translation in the superior cavity (2). Therefore, if the disc is in the normal position, the mouth opening movement generally proceeds almost in a straight line. In contrast, disc displacement interferes with condylar translation, resulting in deviations or deflection of the mouth movement.

Because the path of mouth opening movement has been used to generate data for functional adjustments in the processes of dental prosthetic treatment and TMD screening, various methods have been developed to record and analyze mouth opening movements. The use of mechanical equipment can help to accurately trace the motion, but the disadvantage is that the equipment is heavy and cumbersome, potentially interfering with movement. Another method using an ultrasonic jaw motion analyzer has also been reported $(8,9)$; such devices are increasingly simple, but remain impractical to use in dental clinics. Thus, most clinicians visually evaluate the path of mouth opening movement for TMD screening in a clinical setting. However, a visual examination is subjective, with results that may vary from tester to tester, and the movement path cannot be recorded and quantified. Only a few studies have explored the relationship between patterns of mouth opening movement and disc displacement $(10,11)$.

Recently, artificial intelligence (AI) applications have been actively developed in the dental field $(12,13)$. In this study, an AI-based system including computer vision that analyzes the mouth opening path was developed and the relationship between the path analysis generated by the model and the MRI-based diagnosis was evaluated. We hypothesized that if disc displacement is present, the mouth opening movement would be different from the pattern observed if the disc is in the normal position, and that AIbased systems would be as accurate as clinical experts with extensive clinical experience. The results will be useful for TMD screening by providing quantitative information on patterns of mouth opening movement.

The purpose of this study was to develop an automatic mouth opening tracing system based on computer vision using videos of mouth opening and closing movements, and to investigate the relationship between the path analysis obtained from the developed system and disc position on MRI.

We present the following article in accordance with the MDAR checklist (available at https://dx.doi.org/10.21037/ qims-21-629).

\section{Methods}

\section{Subjects}

The study was conducted in accordance with the Declaration of Helsinki (as revised in 2013). This research was approved by the Institutional Review Board of Yonsei University Dental Hospital (IRB No. 2-2020-0038). Informed consent was obtained from all participants and, if applicable, their legal guardians and patient information 


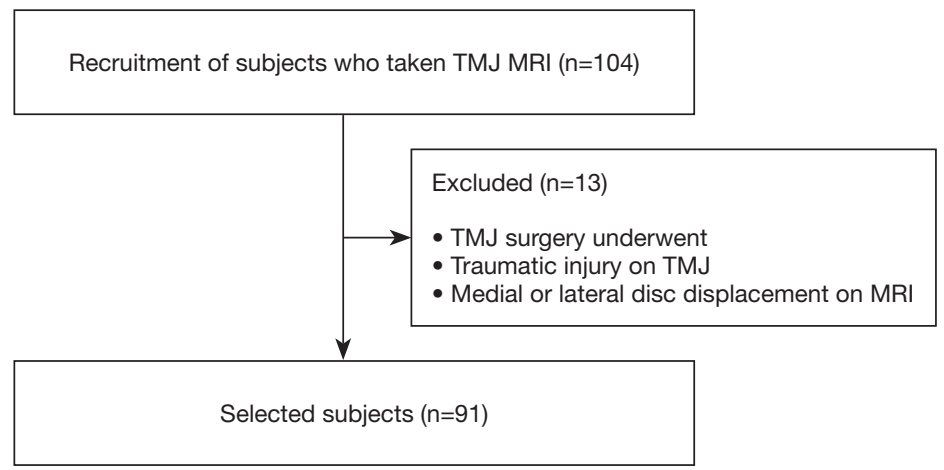

Figure 1 Flow chart diagram of participation. TMJ, temporomandibular joint; MRI, magnetic resonance imaging.

such as age, sex, and patient number were anonymized prior to analysis.

A total of 104 subjects who underwent TMJ MRI examinations at Yonsei University Dental Hospital were selected. Among the subjects, those with a history of TMJ surgery, traumatic injury, and medial or lateral disc displacement on MRI were excluded. Finally, 91 subjects (17 males and 74 females; mean age, 38.42 years old; age range, 13 to 80 years old) were included in this study (Figure 1). All MRI images were obtained according to a routine TMJ protocol in the closed- and open-mouth positions using a SIGNA Pioneer 3.0 T MRI scanner (GE Healthcare, Waukesha, WI, USA) with a 16-channel flex large coil. Sagittal proton density-weighted images were obtained with the following parameters: TE/TR, 50/2,084 ms; bandwidth, $41.67 \mathrm{kHz}$; NEX, 1.5; FOV, $130 \mathrm{~mm} \times 130 \mathrm{~mm}$; slice thickness, $2.5 \mathrm{~mm}$; and scan time, $2 \mathrm{~min} 14 \mathrm{~s}$. Coronal proton density-weighted images were obtained with the following parameters: TE/TR, 50/1,500 ms; bandwidth, $41.67 \mathrm{kHz}$; NEX, 2; FOV, $130 \mathrm{~mm} \times 130 \mathrm{~mm}$; slice thickness, $2.5 \mathrm{~mm}$; and scan time, $2 \mathrm{~min} 3$ s. Sagittal T2-weighted images were obtained with the following parameters: TE/TR, 80/3,088 ms; bandwidth, $25.00 \mathrm{kHz}$; NEX, 1.5; FOV, $130 \mathrm{~mm} \times 130 \mathrm{~mm}$; slice thickness, $2.5 \mathrm{~mm}$; and scan time, $2 \mathrm{~min} 16 \mathrm{~s}$.

On MRI, the normal disc position is one in which the posterior band is located directly superior to the condylar head and the thin intermediate zone is located between the articulating surface in the closed mouth position (14). An oral and maxillofacial radiologist with 15 years of experience assessed the disc position as normal, anterior disc displacement with reduction (DDWR; the posterior band located anterior to the condylar head in the closedmouth position, but with a normal disc-condyle relationship in the open-mouth position), or anterior disc displacement without reduction (DDWOR; the posterior band positioned anterior to the condyle in both the closed- and open-mouth positions). Disc displacement can be unilateral or bilateral. The subjects were divided into six subgroups as follows: (I) group A (normal on both sides), (II) group B (DDWR on only one side), (III) group C (DDWR on both sides), (IV) group D (DDWOR on only one side), (V) group $\mathrm{E}$ (DDWOR on both sides), and (VI) group F (DDWOR on one side and DDWR on the other side). The six subgroups were further grouped as normal, DDWR, or DDWOR. Group $\mathrm{F}$ was included in the DDWOR group. Table 1 shows the general characteristics of the subjects according to groups.

\section{Video acquisition of mouth opening and closing movements}

An 8.0-mm-diameter circular sticker was attached to the center of the subject's upper and lower lips. The subject was seated on a chair with his/her back and head against a wall to minimize body movements during video acquisition. One operator (HS Choi) adjusted the subject's face to ensure that it was not tilted, and the camera was positioned in front of the subject. To remove personally identifiable information, only a limited area from the nose to above the shoulder was taken and the lips were located at the center of the imaging area to reduce camera lens distortion. Before video acquisition, the subject practiced mouth opening and closing movements one to two times under the guidance of an oral radiologist (KJ Jeon). Finally, the subject performed the mouth movements as practiced upon request, and the operator recorded the movements. A digital camera (Sony A5100L; Sony, Minato, Tokyo, Japan) and lens (Sony SELP1650; Sony, Minato, Tokyo, Japan) were used with 
Table 1 Groups of the subjects according to disc position on MRI

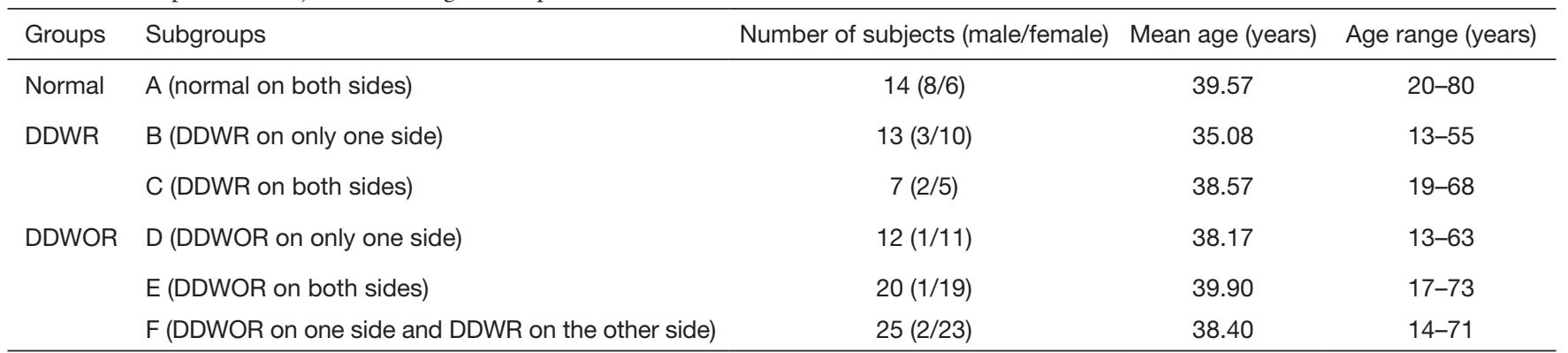

$\mathrm{MRI}$, magnetic resonance imaging; DDWR, anterior disc displacement with reduction; DDWOR, anterior disc displacement without reduction.

the following capture settings: focal length, $16-50 \mathrm{~mm}$ in focal length; aperture range, and f/3.5-5.6; 30 frames per second. The video for input to the model was resized from its original resolution of 1,080 (width) $\times 1,440$ (height) to 540 (width) $\times 720$ (height).

\section{Development of the automated mouth opening tracing system based on computer vision}

A total of 10,604 frames obtained from the videos of 91 subjects were used to develop an automatic tracing system of mouth opening and closing movements. The mouth opening tracing system was developed to automatically output a tracing graph after video input using MATLAB R2019b (MathWorks, Natick, MA, USA). Figure 2 shows the overall process of the developed system. We designed the automated model as follows: (I) development of automated landmark detection of the upper and lower lips from the videos; and (II) graphical visualization of the tracing graph and automatic calculation of the graph height (mouth opening length) and width (sideways values).

In the first step, the landmarks to be automatically detected were defined by the circular stickers attached to the upper and lower lips in each video frame. This process started with inputting the video, and each video frame was converted from an RGB image to a gray-scale image. The model detected the bright circles with a radius of 3-10 (pixels) in each converted frame using the circular Hough transform method. From the two detected circular stickers, the center coordinates ( $\mathrm{x}$ and $\mathrm{y}$ ) and the radius of each circle were extracted. The second step was designed to provide the visual and quantitative information necessary for analysis using automatically detected landmarks. The tracing graph output the lower lip movement based on the upper lip at the same position. In the first frame, the positions of the initially recognized $(x, y)$ coordinates were adjusted to $(0,0)$, and in the subsequent frames, each position of the coordinates was calculated using the equations below.

$\mathrm{x}$-coordinate: (x-coordinate of the lower lip) $-(\mathrm{x}$-coordinate of the upper lip)

y-coordinate: (y-coordinate of the lower lip) -(y-coordinate of the upper lip)

To provide additional information, graph height and width values were calculated from the traced graphs. The computed ( $\mathrm{x}, \mathrm{y})$ coordinates (pixels) using the actual radius of the circular sticker were extracted as the actual length $(\mathrm{mm})$. The height and width were calculated by the following equation.

Height value $=($ maximum $y$-coordinate value $)$

$$
-(\text { minimum y-coordinate value })
$$

Width value $=($ maximum $x$-coordinate value $)$ $-($ minimum $x$-coordinate value $)$

As the final output of the developed automated mouth opening tracing system, a visualized graph with height and width values was presented. The opening tracing was displayed as green and the closing tracing was displayed in orange for easy identification of mouth movement.

\section{Evaluation of the proposed systems and statistical analysis of graphs according to disc position}

For objective evaluation, the expert manually annotated the centers of two circular stickers on 252 randomly selected frames, and this manual annotation data were used as the gold-standard coordinates. To verify the reliability of the proposed system, intraclass correlation coefficients (ICCs), 


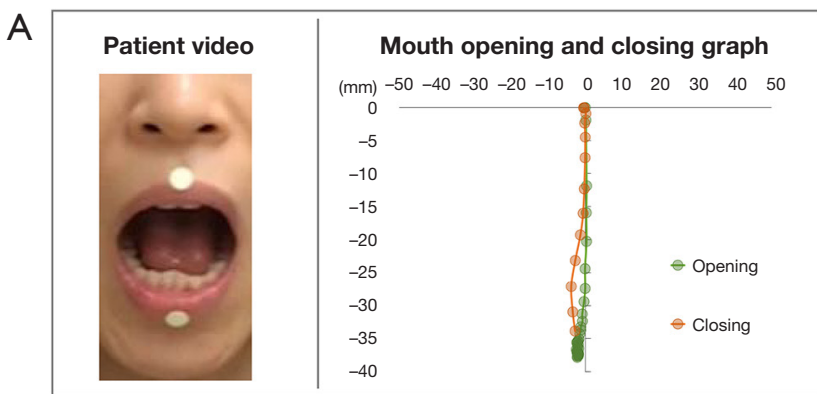

B

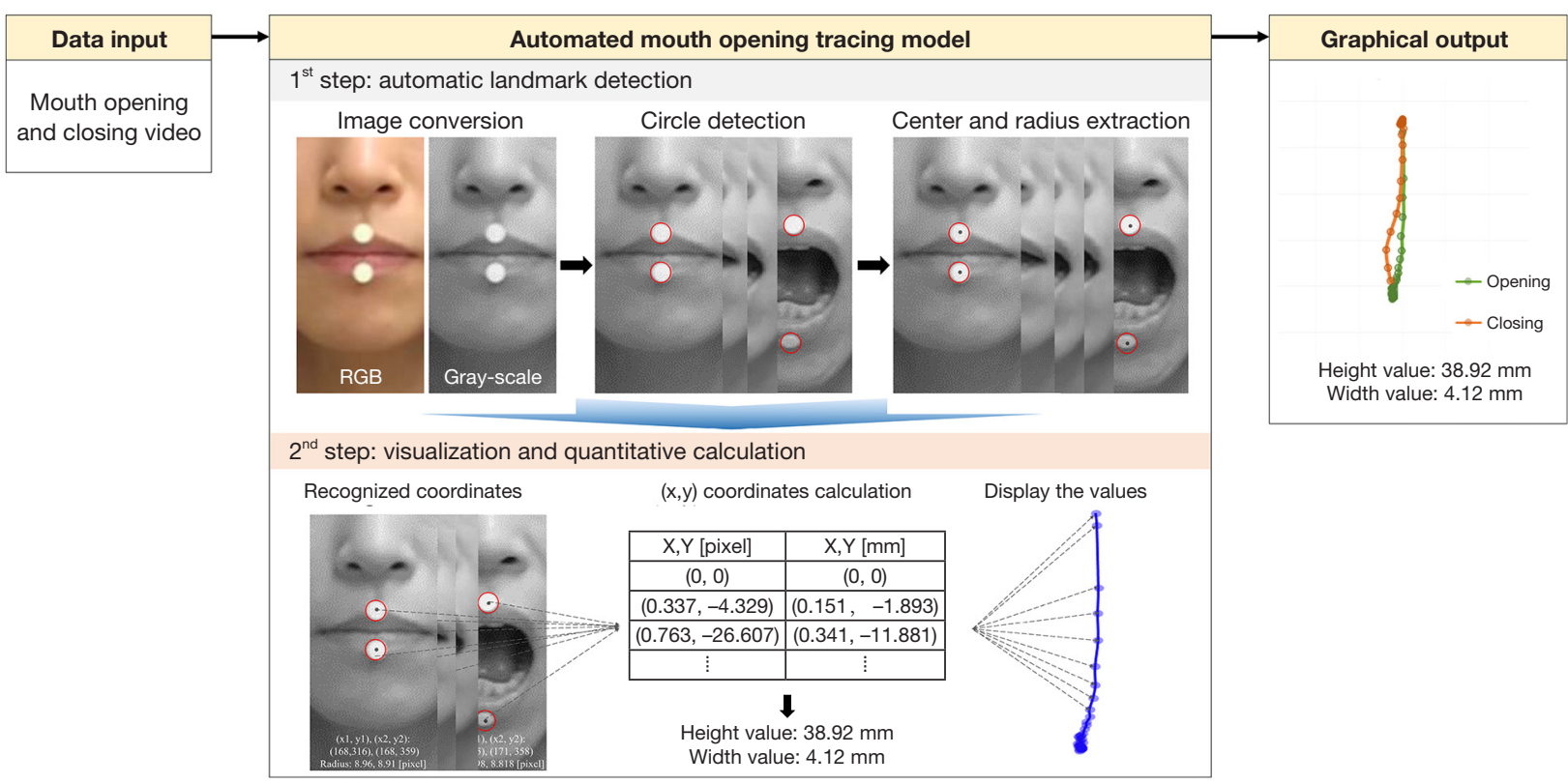

Figure 2 Overall process of our automated mouth opening tracing system. (A) The final output of the proposed system; (B) the mouth opening tracing model and graphical output. This image is published with the patient/participant's consent.

mean absolute error (MAE), and mean squared error (MSE) were calculated between the automatically detected results and the gold-standard values. The automatically detected landmarks showed good reliability, with an ICC of 0.876 , MAE of 0.802 , and MSE of $0.904 \mathrm{~mm}$ compared with the expert's manual annotation.

The graph paths were categorized into three types: straight, sideways-skewed, and limited-straight line graphs. The straight line type displayed alignment from the starting to ending points on the graph during mouth opening and closing movements, and had height values of $35 \mathrm{~mm}$ or more. The sideways-skewed line type had notable sideways changes on the path, such as skews or bouncing with various ranges of height and width values. The limited-straight line type presented a straight line, but with a limited height value of less than $35 \mathrm{~mm}$.

Statistical analysis was performed using the twosided Fisher exact test to evaluate correlations between the three groups (normal, DDWR, and DDWOR) and the proportions of graph path types in each group. The height and width values were presented by descriptive statistics including mean and range. The Shapiro-Wilk and Levene tests were conducted to assess the normality of the distribution and the equality of variance of the data according to group. Statistically significant differences in height and width were calculated using Welch's one-way analysis of variance (ANOVA) with the post-hoc test using the Games-Howell multiple comparison test with a $5 \%$ significance level. IBM SPSS Statistics version 25.0 (IBM Corp., Armonk, NY, USA) was used for statistical analysis. 
Table 2 Graph path types, height, and width of automated mouth opening tracing results according to groups

\begin{tabular}{|c|c|c|c|c|c|c|}
\hline Groups & Subgroups & \multicolumn{3}{|c|}{ Graph path types, n (\%) } & Height range (mean) & Width range (mean) \\
\hline Normal & $A$ & $12(85.72)$ & $1(7.14)$ & $1(7.14)$ & $25.86-64.48(44.90)$ & $1.41-6.41(3.76)$ \\
\hline \multirow[t]{2}{*}{ DDWR } & $\mathrm{B}$ & $1(7.70)$ & $10(76.92)$ & $2(15.38)$ & $27.83-59.49$ (38.60) & $1.21-9.53(4.60)$ \\
\hline & $\mathrm{C}$ & $2(28.57)$ & $5(71.43)$ & $0(0)$ & $30.8-53.52$ (38.31) & $2.83-8.96$ (4.73) \\
\hline \multirow{2}{*}{ DDWOR } & $E$ & $2(10.00)$ & $13(65.00)$ & $5(25.00)$ & $20.89-55.19$ (34.58) & $1.35-7.18$ (3.72) \\
\hline & $\mathrm{F}$ & $2(8.00)$ & $19(76.00)$ & $4(16.00)$ & $24.37-56.66$ (36.73) & $1.19-10.17$ (4.23) \\
\hline
\end{tabular}

The unit of height and width values are millimeters $(\mathrm{mm})$. DDWR, anterior disc displacement with reduction; DDWOR, anterior disc displacement without reduction.

Table 3 Distribution of graph path types between the normal, DDWR, and DDWOR groups

\begin{tabular}{|c|c|c|c|c|}
\hline Groups & \multicolumn{3}{|c|}{ Graph path types, n (\%) } & Total \\
\hline Normal & $12(85.72)$ & $1(7.14)$ & $1(7.14)$ & 14 \\
\hline DDWR & $3(15.00)$ & $15(75.00)$ & $2(10.00)$ & 20 \\
\hline DDWOR & $6(10.53)$ & 39 (68.42) & $12(21.05)$ & 57 \\
\hline
\end{tabular}

Two-sided Fisher's exact test, ${ }^{*} \mathrm{P}<0.05$. DDWR, anterior disc displacement with reduction; DDWOR, anterior disc displacement without reduction.

\section{Results}

In group A, $85.72 \%$ of the graph paths were straight line graphs, while sideways-skewed line graphs were present in $76.92 \%, 71.43 \%, 58.33 \%, 65 \%$, and $76 \%$ of subjects in groups B to F, respectively. The limited-straight line pattern was found in $25 \%$ of subjects in group $\mathrm{E}$. The mean height of the graphs was $44.90 \mathrm{~mm}$ in group A and $34.58 \mathrm{~mm}$ in group E (Table 2).

A correlation between the disc position on MRI and graph path type was statistically confirmed in the three groups $(\mathrm{P}<0.001)$ (Table 3). The straight line type was present in $85.72 \%$ of subjects in the normal group, while the sideways-skewed and limited-straight line types were found in $85.0 \%$ and $89.47 \%$ of subjects in the DDWR and DDWOR groups, respectively (Figure 3).

Figure 4 shows a comparison of the height of mouth opening among groups. The mean height in the DDWOR group was $35.78 \pm 10.24 \mathrm{~mm}$, which was lower than that of the normal group $(44.90 \pm 9.61 \mathrm{~mm})$ and the DDWR group $(38.50 \pm 8.92 \mathrm{~mm})$, reflecting a statistically significant difference $(\mathrm{P}<0.05)$. No statistically significant difference in the width of mouth opening was found among the groups $(\mathrm{P}>0.05)$.

\section{Discussion}

Our study showed a correlation between the graph paths identified using the developed system and the disc position on MRI. Approximately one-third of the population has TMD, which is a chronic disease that may not be diagnosed until mouth opening limitation or pain develops $(1,2)$. TMD is a very complex condition, and its treatment requires an interdisciplinary approach $(15,16)$. Anterior disc displacement is considered to be one of the main causes of TMD (17). MRI can provide accurate diagnostic information on disc displacement, but its use is limited due to cost, time, and accessibility for most dental clinicians. Thus, clinical examinations for disc displacement diagnosis include pain with mandibular function, abnormal mandibular movement, and joint sounds (click, popping, crepitus, and thud sensation) (18). 

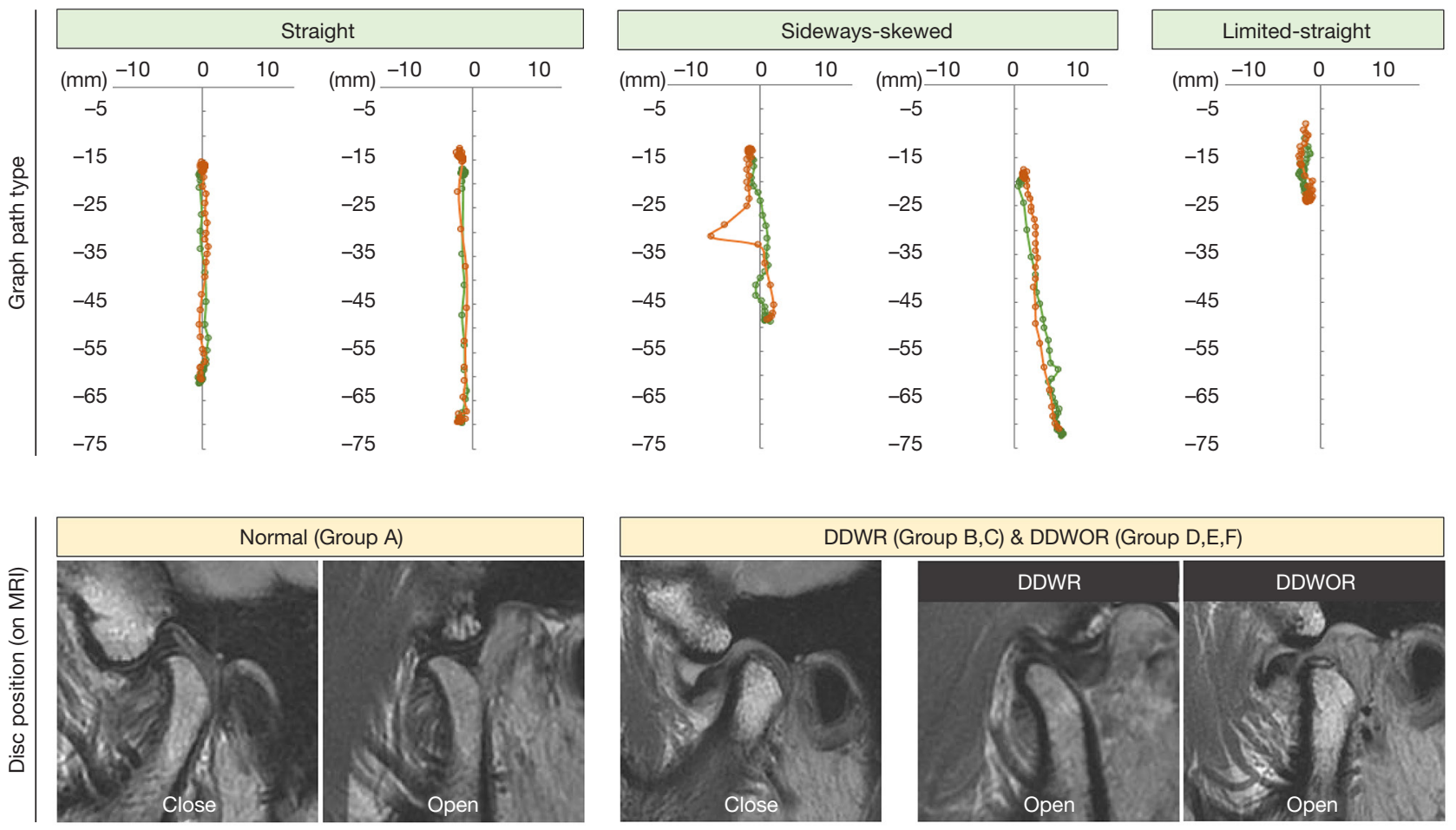

Figure 3 Different types of mouth opening tracing from the proposed automated system and their relationships with the disc position on MRI. In general, the normal group showed the straight line type, and DDWR and DDWOR groups showed the sideways-skewed or limited-straight line type. MRI, magnetic resonance imaging. This image is published with the patient/participant's consent.

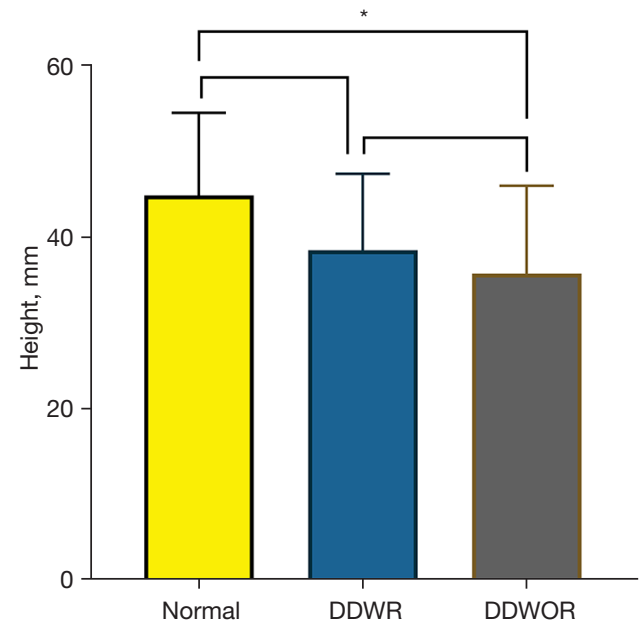

Figure 4 Comparison of height obtained from the automated mouth opening tracing system according to groups classified by disc position. Welch's analysis of variance and the Games-Howell post hoc test were used for the statistical analysis $\left({ }^{*}, \mathrm{P}<0.05\right)$.
Pain is divided into TMJ and masticatory muscle pain, but it is not easy to accurately distinguish between them (17). Joint sounds are caused by disc displacement and/or osteoarthritis, but it is difficult to diagnose osteoarthritis without computed tomography or cone-beam computed tomography (19-21). Abnormal mandibular movements include deviation, deflection, and limitation of mouth opening. During the mouth opening and closing movement, deflection refers to visible deviation to one side at the maximum opening. Deviation is defined as visible left or right movement during opening that returns to the midline before or near the maximum opening. Limitation of mouth opening refers to an unassisted maximum opening of less than $35 \mathrm{~mm}$ according to the Diagnostic Criteria for Temporomandibular Disorders (17). Limitation of mouth opening can be assessed as the maximum amount of opening measured by the distance between the incisal edges, and there are two types: acute and chronic. If limitation of mouth opening is due to an acute lock, the patient is likely to recognize that there is a problem; however, if TMD is chronic and the disease progresses gradually, 
the patient may not recognize that the mouth opening is restricted. Approximately $3.6 \%$ to $7 \%$ of TMD patients seek professional care for pain, sound, and limitation of mouth opening, and many patients leave without treatment $(1,2)$. As a clinical examination, mouth opening and closing movement can be objectified and quantified, and deviation or deflection can be graphed by recording mouth opening and closing movements using special equipment. Recording devices are expensive to purchase, are not often used due to the time cost, weight, and discomfort of the device, and rely on a subjective visual inspection. Since a visual examination has the drawback that it cannot be visualized and quantified, it would be very useful if visualization and quantification could be performed using only a video of mouth opening and closing movement without any special equipment.

$\mathrm{AI}$ is used in various dental fields such as dental caries diagnosis (22-24), the diagnosis of oral diseases such as squamous cell carcinoma (25), the formulation of orthodontic treatment plans (26-28), and assessments of the developmental stages of third molars (29). There have been few studies using AI for TMD; therefore, in this study, we developed an $\mathrm{AI}$ system based on computer vision to trace the mouth opening path using only mouth opening and closing videos. In this novel study of mouth opening tracing, circular stickers were attached to the upper and lower lips to recognize the landmarks. Previously, paths and height have been analyzed at interincisal points of the maxilla and mandible. However, since the anterior teeth are not clearly visible in the mouth opening and closing videos, we analyzed movement based on the center points of the stickers on the upper and lower lips. The automated mouth opening tracing system developed in this study was objectively reliable.

In this study, patients with a normal disc position mostly showed the straight line type, while those with anterior disc displacement showed the sideways-skewed or limitedstraight line type. In the normal group, more than $85 \%$ of subjects showed the straight line type, and only two did not. One of the subjects showed the sideways-skewed type with left deviation, but a neurological problem was suspected and the patient was referred to a neurologist. The other one showed the limited-straight line type. That patient had masseter muscle pain and a history of Botox injections, reflecting muscle problems, not disc displacement. In the group with anterior disc displacement, only about $12 \%$ of patients showed the straight line type. Of the nine patients with anterior disc displacement who presented the straight line type, the DDWR joints showed slight or partial disc displacement in the early stage. In the DDWOR joints, the disc displacement was in the late stage, and the disc was not stuck until the maximum opening, or the disc located in front at the condylar head moved with the condylar head at the time of opening. The proposed automated mouth opening tracing system detected anterior disc displacement well, but it seemed likely to miss detection of initial and late stages of disc displacement.

The height of mouth opening was highest in the normal group, followed in descending order by the DDWR and DDWOR groups, and the difference was statistically significant between the normal and DDWOR groups. The width was lowest in the normal group, followed in increasing order by the DDWOR and DDWR groups, but the difference showed no statistical significance. The reason why DDWOR group had narrower movements than the DDWR group is thought to be that the condyle was stuck in the disc and limitation of mouth opening occurred before the sideways motion.

The main limitations of this study are that each group, especially the normal group, had a small number of subjects, the overall age range was very wide, and the number of male participants was small. With a large number of samples, we will be able to better characterize the mouth opening graphs according to disc position. In addition, potential measurement errors can occur due to the omission of lens calibration and posterior movement of the lower lip upon opening of the mouth. Although we constructed a controlled experimental environment, with steps such as locating the mouth at the center of the image area and fixing the subject's posture, technical improvements may be necessary to obtain more accurate results. Further research may make it possible to trace paths in real-time without circular stickers by advances in $\mathrm{AI}$ or facial landmark detection using computer vision.

\section{Conclusions}

The developed mouth opening tracing system based on computer vision was reliable and its results were correlated with disc position on MRI. The proposed system provided objective and quantitative information about the different trajectories of mouth opening and closing movements in people with disc displacement or a normal disc position. This will help clinicians screen TMD and will facilitate precise diagnoses for patients.

\section{Acknowledgments}

Funding: This work was supported by a National Research 
Foundation of Korea (NRF) grant funded by the Korean government (MSIT) (2019R1A2C1007508).

\section{Footnote}

Reporting Checklist: The authors have completed the MDAR checklist. Available at https://dx.doi.org/10.21037/qims-21629

Conflicts of Interest: All authors have completed the ICMJE uniform disclosure form (available at https://dx.doi. org/10.21037/qims-21-629). The authors have no conflicts of interest to declare.

Ethical Statement: The authors are accountable for all aspects of the work in ensuring that questions related to the accuracy or integrity of any part of the work are appropriately investigated and resolved. The study was conducted in accordance with the Declaration of Helsinki (as revised in 2013). This research was approved by the Institutional Review Board of Yonsei University Dental Hospital (No. 2-2020-0038). Informed consent was obtained from all participants and, if applicable, their legal guardians.

Open Access Statement: This is an Open Access article distributed in accordance with the Creative Commons Attribution-NonCommercial-NoDerivs 4.0 International License (CC BY-NC-ND 4.0), which permits the noncommercial replication and distribution of the article with the strict proviso that no changes or edits are made and the original work is properly cited (including links to both the formal publication through the relevant DOI and the license). See: https://creativecommons.org/licenses/by-nc-nd/4.0/.

\section{References}

1. de Leeuw R. American Academy of Orofacial Pain. Orofacial Pain: Guidelines for Assessment, Diagnosis, and Management. Chicago: Quintessence Publishing Co., Inc., 2008.

2. Okeson JP. Management of temporomandibular disorders and occlusion. London: Mosby, 2020.

3. Yang HY, Kim ME. Prevalence and Treatment Pattern of Korean Patients with Temporomandibular Disorders. J Oral Med Pain 2009;34:63-79.

4. Larheim TA. Role of magnetic resonance imaging in the clinical diagnosis of the temporomandibular joint. Cells
Tissues Organs 2005;180:6-21.

5. Emshoff R, Rudisch A, Innerhofer K, Brandlmaier I, Moschen I, Bertram S. Magnetic resonance imaging findings of internal derangement in temporomandibular joints without a clinical diagnosis of temporomandibular disorder. J Oral Rehabil 2002;29:516-22.

6. Jeon KJ, Lee C, Choi YJ, Han SS. Analysis of threedimensional imaging findings and clinical symptoms in patients with temporomandibular joint disorders. Quant Imaging Med Surg 2021;11:1921-31.

7. Yatani H, Sonoyama W, Kuboki T, Matsuka Y, Orsini MG, Yamashita A. The validity of clinical examination for diagnosing anterior disk displacement with reduction. Oral Surg Oral Med Oral Pathol Oral Radiol Endod 1998;85:647-53.

8. Kordass B, Hugger A, Bernhardt O. Correlation between computer-assisted measurements of mandibular opening and closing movements and clinical symptoms of temporomandibular dysfunction. Int J Comput Dent 2012;15:93-107.

9. Ahlers MO, Bernhardt O, Jakstat HA, Kordaß B, Türp JC, Schindler HJ, Hugger A. Motion analysis of the mandible: guidelines for standardized analysis of computer-assisted recording of condylar movements. Int J Comput Dent 2015;18:201-23.

10. Kim HW, Shin SS, Kim JS, Kim KY, Kim YJ, Hong SM, Cheon SH, Park YH, Choi WC, Park JW. Evaluation of clinical methods in the diagnosis of temporomandibular joint disorders: a comparison study with magnetic resonance imaging. J Korean Assoc Oral Maxillofac Surg 2007;33:367-74.

11. Alajbeg IZ, Gikić M, Valentić-Peruzović M. Mandibular Range of Movement and Pain Intensity in Patients with Anterior Disc Displacement without Reduction. Acta Stomatol Croat 2015;49:119-27.

12. Hung K, Montalvao C, Tanaka R, Kawai T, Bornstein $\mathrm{MM}$. The use and performance of artificial intelligence applications in dental and maxillofacial radiology: A systematic review. Dentomaxillofac Radiol 2020;49:20190107.

13. Khanagar SB, Al-Ehaideb A, Maganur PC, Vishwanathaiah S, Patil S, Baeshen HA, Sarode SC, Bhandi S.

Developments, application, and performance of artificial intelligence in dentistry - A systematic review. J Dent Sci 2021;16:508-22.

14. Koh KJ, Park HN, Kim KA. Relationship between anterior disc displacement with/without reduction and effusion in temporomandibular disorder patients using magnetic 
resonance imaging. Imaging Sci Dent 2013;43:245-51.

15. Derwich M, Mitus-Kenig M, Pawlowska E. Interdisciplinary Approach to the Temporomandibular Joint Osteoarthritis-Review of the Literature. Medicina (Kaunas) 2020;56:225.

16. Derwich M, Mitus-Kenig M, Pawlowska E. Orally Administered NSAIDs-General Characteristics and Usage in the Treatment of Temporomandibular Joint Osteoarthritis-A Narrative Review. Pharmaceuticals (Basel) 2021;14:219.

17. Schiffman E, Ohrbach R, Truelove E, Look J, Anderson G, Goulet JP, et al. Diagnostic Criteria for Temporomandibular Disorders (DC/TMD) for Clinical and Research Applications: recommendations of the International RDC/TMD Consortium Network* and Orofacial Pain Special Interest Group†. J Oral Facial Pain Headache 2014;28:6-27.

18. Derwich M, Mitus-Kenig M, Pawlowska E. Is the Temporomandibular Joints' Reciprocal Clicking Related to the Morphology and Position of the Mandible, as Well as to the Sagittal Position of Lower Incisors?-A Case-Control Study. Int J Environ Res Public Health 2021;18:4994.

19. dos Anjos Pontual ML, Freire JS, Barbosa JM, Frazão MA, dos Anjos Pontual A. Evaluation of bone changes in the temporomandibular joint using cone beam CT. Dentomaxillofac Radiol 2012;41:24-9.

20. Derwich M, Mitus-Kenig M, Pawlowska E. Temporomandibular Joints' Morphology and Osteoarthritic Changes in Cone-Beam Computed Tomography Images in Patients with and without Reciprocal Clicking-A Case Control Study. Int J Environ Res Public Health 2020;17:3428.

21. Derwich M, Mitus-Kenig M, Pawlowska E. Morphology of the Temporomandibular Joints Regarding the Presence of Osteoarthritic Changes. Int J Environ Res Public Health 2020;17:2923.

22. Devito KL, de Souza Barbosa F, Felippe Filho WN. An artificial multilayer perceptron neural network for diagnosis of proximal dental caries. Oral Surg Oral Med Oral Pathol Oral Radiol Endod 2008;106:879-84.

23. Lee JH, Kim DH, Jeong SN, Choi SH. Detection and diagnosis of dental caries using a deep learningbased convolutional neural network algorithm. J Dent 2018;77:106-11.

24. Hung M, Voss MW, Rosales MN, Li W, Su W, Xu J, Bounsanga J, Ruiz-Negrón B, Lauren E, Licari FW. Application of machine learning for diagnostic prediction of root caries. Gerodontology 2019;36:395-404.

25. Aubreville M, Knipfer C, Oetter N, Jaremenko C, Rodner E, Denzler J, Bohr C, Neumann H, Stelzle F, Maier A. Automatic Classification of Cancerous Tissue in Laserendomicroscopy Images of the Oral Cavity using Deep Learning. Sci Rep 2017;7:11979.

26. Xie X, Wang L, Wang A. Artificial neural network modeling for deciding if extractions are necessary prior to orthodontic treatment. Angle Orthod 2010;80:262-6.

27. Jung SK, Kim TW. New approach for the diagnosis of extractions with neural network machine learning. Am J Orthod Dentofacial Orthop 2016;149:127-33.

28. Choi HI, Jung SK, Baek SH, Lim WH, Ahn SJ, Yang IH, Kim TW. Artificial Intelligent Model With Neural Network Machine Learning for the Diagnosis of Orthognathic Surgery. J Craniofac Surg 2019;30:1986-9.

29. De Tobel J, Radesh P, Vandermeulen D, Thevissen PW. An automated technique to stage lower third molar development on panoramic radiographs for age estimation: a pilot study. J Forensic Odontostomatol 2017;35:42-54.

Cite this article as: Jeon KJ, Kim YH, Ha EG, Choi HS, Ahn HJ, Lee JR, Hwang D, Han SS. Quantitative analysis of the mouth opening movement of temporomandibular joint disorder patients according to disc position using computer vision: a pilot study. Quant Imaging Med Surg 2022;12(3):19091918. doi: 10.21037/qims-21-629 